Case Report

OOpen Access

A) CrossMark

\title{
One-and-a-half syndrome associated with peripheral facial nerve palsy after pontine ischaemic stroke
}

Keywords: acute stroke, lower motor neuron facial palsy, conjugate gaze palsy, eight-and-a-half syndrome, ischaemic stroke, one-and-a-half syndrome

\section{Introduction}

A 63-year-old man with a sudden facial paralysis, diplopia, and ophthalmoplegia was admitted at an emergency unit. The neurological examination revealed left peripheral facial palsy, conjugated complete horizontal palsy for left gaze, as well as for adduction in the left eye to the right gaze, with normal pupillary reflexes and conjugated vertical upward and downward gaze on saccadic and smooth pursuit eye movements (Figure 1). A head computerized tomography had no acute ischaemic signs, and he was discharged. The brain magnetic resonance performed five days after the ictus showed a defined left paramedian tegmental dorsal pontine infarct (Figure 2). The association between the one-and-a-half syndrome and the ipsilateral lower motor neurontype facial palsy is called eight-and-a-half syndrome ${ }^{1}-$ a finding that can be understood when the brainstem anatomy is analyzed.

\author{
Volume 7 Issue 2 - 2020 \\ Louise Makarem Oliveira,' Pablo Vinicius \\ Silveira Feitoza ${ }^{2}$ \\ 'Student of Medicine, School of Medicine, Federal University of \\ Amazonas (UFAM), Brazil \\ ${ }^{2}$ Doctor of Medicine (Neurology), Department of Neurology, \\ Division of Neurology, School of Medicine, Federal University of \\ Amazonas (UFAM), Brazil
}

Correspondence: Pablo Vinicius Silveira Feitoza, Division of Neurology, School of Medicine, Federal University of Amazonas (UFAM). 1053, Afonso Pena St.- Centro, 69020-160, Manaus, Amazonas, Brazil, Email pvsfeitoza@ufam.edu.br

Received: March 08, 2020 | Published: April 29, 2020

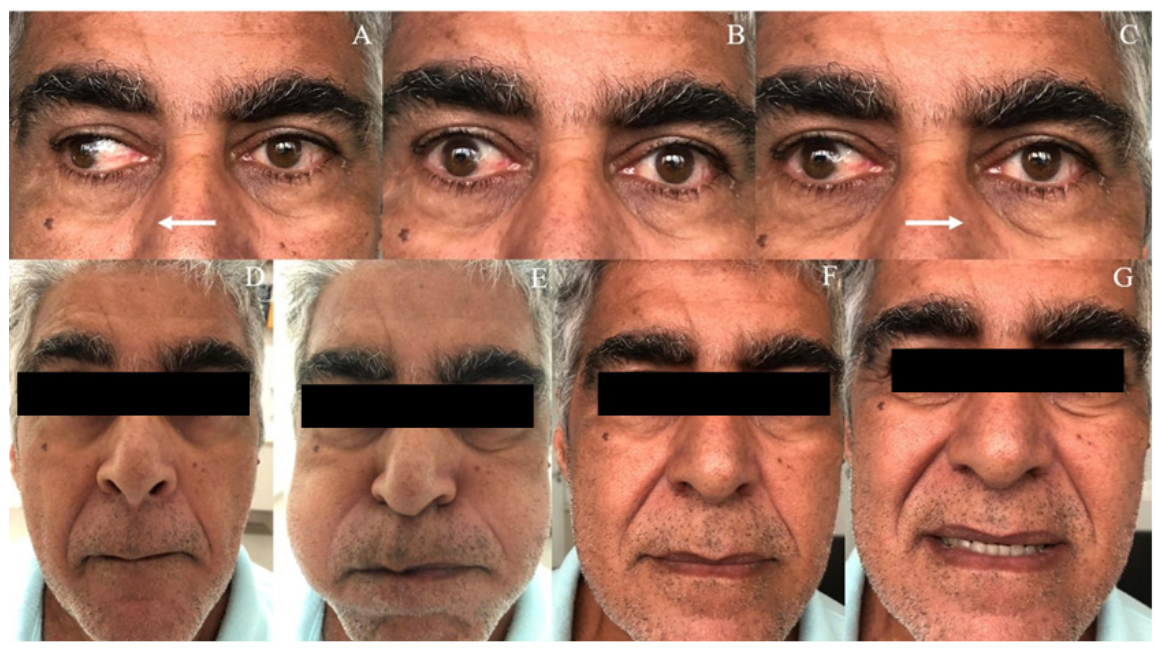

Figure I Positions of gaze and facial motricity. (A) Right gaze with adduction paresis of left eye. (B) Forward gaze in primary position. (C) Left gaze, with a conjugate palsy to this side. (D) Preserved upward gaze and asymmetrical forehead wrinkles. (E) Left buccinator muscle weakness. (F) Flattened nasolabial fold on the left side. (G) Asymmetrical smile.

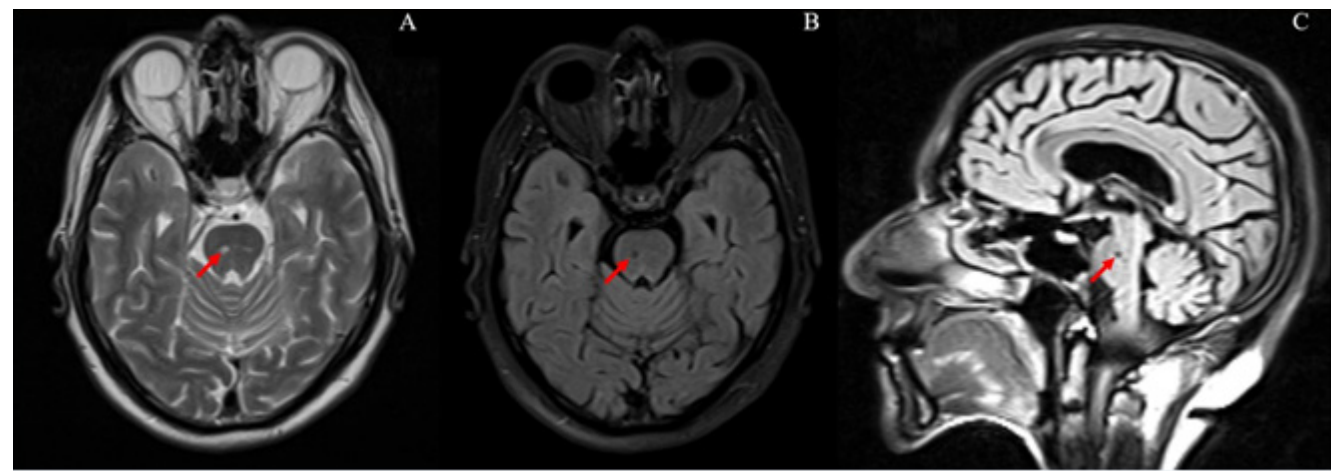

Figure 2 Magnetic resonance images revealing pontine infarction (arrow). (A) Axial T2. (B) Axial T2 FLAIR. (C) Sagital T2 FLAIR. 
The horizontal gaze results from the contraction of both the medial and the lateral rectus of different eyes, which are respectively, controlled by the oculomotor (IIIn) and abducens (VIn) nuclei. The medial longitudinal fasciculus (MLF) is responsible for connecting not only these but also the vestibular and trochlear nuclei, allowing conjugated eye movement. According to this circuit, the abducens nucleus ipsilateral to the direction of the gaze acts as a "movement center," projecting fibers directly to the corresponding lateral rectus muscle and, through MLF, to the contralateral IIIn, which in turn stimulates the medial rectus muscle contraction. ${ }^{2}$ Another essential structure for horizontal gaze lies in the pontine tegmentum: the paramedian pontine reticular formation (PPRF), which interconnects the cortical inputs to the abducens nucleus. The "one-and-a-half" syndrome, ${ }^{3,4}$ defined as a conjugate palsy to one side, associated with impaired adduction to the other side, is explained by a lesion that involves the MLF and either the adjacent PPRF or abducens nucleus on the side of the complete gaze palsy. The abduction of the eye contralateral to the lesion, as well as the vertical gaze and the convergence, is spared.
A

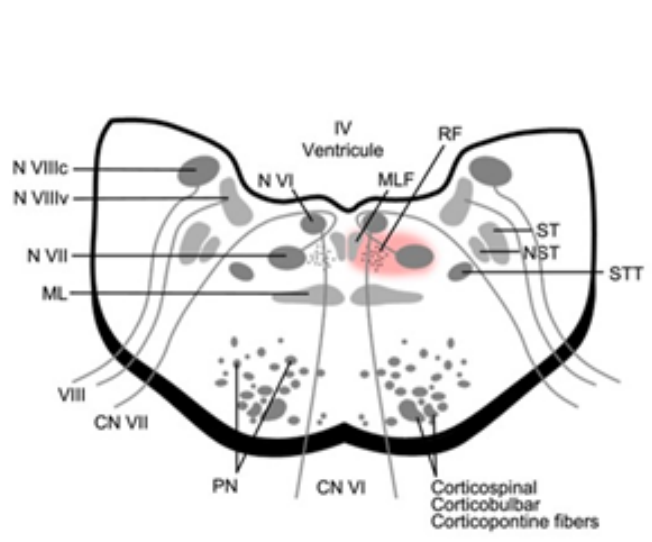

The distinction between the PPRF or the VIn involvement can be clinically made, by evaluating the rapid eyes movements: quick phases of nystagmus and saccades. ${ }^{5}$ When the rostral part of the PPRF is injured, there is an absence of all rapid movements ipsilateral to the lesion alongside preserved ipsilateral vestibulo-ocular response. On the other hand, if the abducens nucleus is the jeopardized site, nondissociated ipsilateral horizontal gaze palsy can be noticed, with the loss of vestibulo-ocular reflexes. ${ }^{5}$

Peripheral facial nerve palsy is caused by a lower motor neuron lesion, ${ }^{1}$ and affects the entire ipsilateral hemiface. The facial nerve nucleus (VIIn) is situated ventrolaterally on the pons, caudally to the trigeminal motor nucleus and lateral to the PPRF. Its fibers run dorsomedially towards the fourth ventricle and loop around the VIn. The proximity between these structures explains why injuries on the VIn are often accompanied by peripheral facial palsy (Figure 3). Our patient had a classical eight-and-a-half syndrome due to an ischaemic stroke on the dorsal pons. It shows the complexity of supranuclear eye movement control and the intrinsic relationship among the MRF nuclei.
B

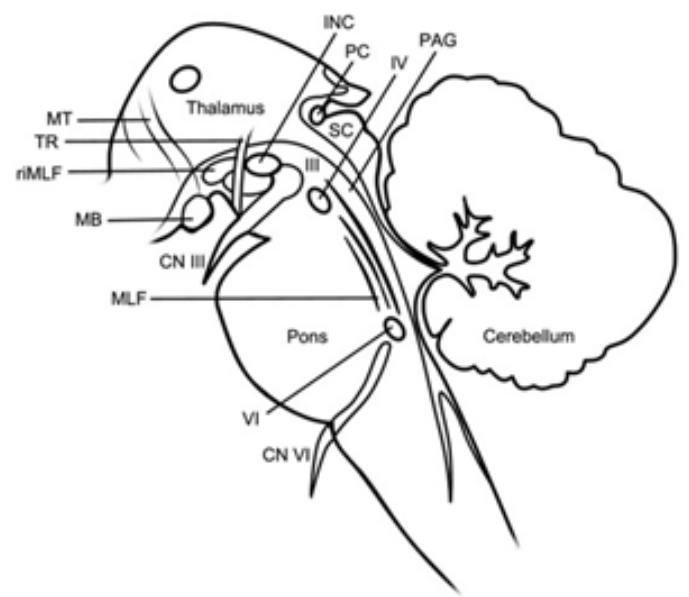

Figure 3 Anatomic schemes of the nuclei related to horizontal gaze and to facial motor control. (A) Axial view of the pons. The red-colored area indicates the presumed site of lesion. RF, reticular formation; MLF, medial longitudinal fascicle; STT, spinothalamic tract; NST, nucleus of spinal tract (V); ST, spinal tract (V); PN, pontine nuclei; CN VI, cranial nerve - abducens (VI); CN VII, cranial nerve - facial (VII); ML, medial lemniscus; NVIIlc, cochlear nucleus; NVIIlv, vestibular nuclei. (B) Sagital view of the brainsteam. MT: mammillothalamic tract; TR, tractus retroflexus; MB, mamillary bodies; riMLF, rostral interstitial nucleus of the medial longitudinal fascicle; INC, interstitial nucleus of Cajal; PC, posterior comissure; SC, superior colliculus; IV, nucleus of cranial nerve trochlear (IV); PAG, periaquedutal grey; CNVI, cranial nerve - abducens (VI); MLF, medial longitudinal fascicle; CN III, cranial nerve - oculomotor (III).

\section{Acknowledgments}

Louise Makarem Oliveira: Study concept and design, reports no disclosures.

Pablo Vinicius Silveira Feitoza: Study concept and design, acquisition of data, reports no disclosures.

\section{Funding}

No funds were required.

\section{Conflicts of interest}

We declare that the article is original and unpublished and has not been submitted to any other periodical. We also express our consent in relation to the submission process.

\section{References}

1. Eggenberger EJ. Eight-and-a-half syndrome: one-and-a-half syndrome plus cranial nerve VII palsy. Neuroophthalmology. 1998;18(2):114-116.

2. Büttner-Ennever JA, Horn AKE. Reticular formation: eye movements, gaze and blinks. In: Paxinos G, Mai JK, editors. The human nervous system. San Diego, CA: Elsevier Academic Press; 2004. p. 479-510.

3. Pierrot-Deseilligny C, Chain F, Serdaru M, et al. The "one-and-a-half" syndrome. Electro-oculographic analyses of five cases with deductions about the physiological mechanisms of lateral gaze. Brain. 1981;104:665699.

4. Wall M, Wray SH. The one-and-a-half syndrome. A unilateral disorder of the pontine tegmentum: a study of 20 cases and review of the literature. Neurology. 1983;33:971-980.

5. Deleu D, Solheid C, Michotte A, et al. Dissociated ipsilateral horizontal gaze palsy in one-and-a-half syndrome: a clinicopathologic study. Neurology. 1988;38:1278-1280. 